\title{
Improving weak-signal identification via predetection background suppression by a pixel-level, surface-wave enabled dark-field aperture
}

\author{
Guoan Zheng ${ }^{1, *}$ and Changhuei Yang ${ }^{1,2}$ \\ ${ }^{1}$ Department of Electrical Engineering, California Institute of Technology, Pasadena, California 91125, USA \\ ${ }^{2}$ Department of Bioengineering, California Institute of Technology, Pasadena, California 91125, USA \\ *Corresponding author: gazheng@caltech.edu
}

Received April 13, 2010; revised June 24, 2010; accepted July 4, 2010;

posted July 13, 2010 (Doc. ID 126951); published July 29, 2010

\begin{abstract}
We report the successful implementation of a surface-wave enabled dark-field aperture (SWEDA) directly on a complementary metal-oxide semiconductor sensor pixel $(2.2 \mu \mathrm{m})$. This SWEDA pixel allows predetection cancellation of a uniform coherent background. We show that the signal-to-noise ratio (SNR) of the SWEDA pixel is better than that of a single undressed pixel over a significant range of signal-to-background ratio (SBR). For a small SBR value $\left(\mathrm{SBR}=0.001\right.$, background intensity $=3.96 \mathrm{~W} / \mathrm{m}^{2}$, integration time $\left.=5 \mathrm{~ms}\right)$, we further demonstrate that a SWEDA pixel can detect a weak localized signal buried in a high background, while conventional postdetection background subtraction cannot (improved SNR $=2.2$ versus SNR = 0.26). (C) 2010 Optical Society of America

OCIS codes: $240.6680,250.0040,250.5403,120.3180$.
\end{abstract}

Discernment of a weak signal in the presence of a high background is a relatively common optical problem [1]. Interestingly, the wave nature of light implies that predetection background cancellation can result in more sensitive signal detection than postdetection background cancellation. Specifically, consider the scenario in which we have a weak and spatially localized optical light spot (signal) hidden within a spatially dispersed uniform coherent background. We can attempt to detect this signal by making simultaneous measurements of the total light field at the proximal location of the signal light spot, and the total light field at an off location. We can then attempt to numerically cancel the background by subtracting the second measurement from the first. Unfortunately, this subtraction would not be perfect, as the measurements would inevitably contain detection-associated noise terms - at the least, we would have shot noise. Our ability to identify the signal requires that the signal be larger than the detection noise terms. This problem is a fundamental one-if we are given perfect shot-noise-limited detectors and a perfect uniform background, there would still exist a lower fundamental limit to our signal detection ability. The signal-to-noise ratio (SNR) of such a postdetection background subtraction scheme is defined by

$$
\begin{aligned}
\mathrm{SNR}_{\text {post }}= & \frac{E_{\text {signal }}}{E_{\text {noise }}+E_{\text {background fluctuation }}} \\
& \times \sqrt{\frac{\varepsilon \tau P_{\text {signal }}}{h \nu}} \frac{\sqrt{\mathrm{SBR}}}{\sqrt{\mathrm{SBR}}+1}
\end{aligned}
$$

where $E_{\text {signal }}, E_{\text {noise }}$, and $E_{\text {background fluctuation }}$ are the energy of signal, noise, and background fluctuation; $P_{\text {signal }}$ is the power of the signal; $\varepsilon$ is the detector quantum efficiency; $\tau$ is the integration time; and SBR is the signal-tobackground ratio. On the other hand, if we are able to optically cancel the background prior to detection, the process of background photons actualizing on the detectors would not occur (conversion of light from wave to statistical particle nature) and we would not have to contend with detection-associated background noise terms. In this case, our signal detection capability would be identical to the case where the relevant optical signal is present without the background. Mathematically, the counterpart SNR equation for this predetection background subtraction scheme is given by

$$
\begin{aligned}
\mathrm{SNR}_{\text {post }}= & \frac{E_{\text {signal }}}{E_{\text {noise }}+E_{\text {background fluctuation }}} \text { detector shot noise limit } \\
& \times \sqrt{\frac{\varepsilon \tau P_{\text {signal }}}{h \nu}} \frac{\sqrt{\mathrm{SBR}}}{\sqrt{\mathrm{SBR}}+\sqrt{1 / \delta_{b}}} \stackrel{\delta_{b} \gg 1}{\rightarrow} \sqrt{\frac{\varepsilon \tau P_{\text {signal }}}{h \nu}},(2)
\end{aligned}
$$

where $\delta_{b}$ is the suppression ratio of the background.

In the past few years, there has been an intense interest in designing a new compact optical structure for improving the performance of a photodetector or an image sensor [2,3]. In this Letter, we report the successful implementation of a modified complementary metal-oxide semiconductor (CMOS) sensor pixel that is capable of accomplishing predetection background cancellation. This work builds upon our recent demonstration of a background suppression structure, termed the surfacewave enabled dark-field aperture (SWEDA). In that work, we showed that a SWEDA can transmit light with a higher image contrast (SBR) when compared with an undressed hole [4]. However, it is not clear that a sensor pixel patterned with a SWEDA would improve the noise performance (SNR) compared with an undressed sensor pixel. An undressed pixel would be able to collect more signal light than a SWEDA pixel, as the SWEDA transmissive area is small, but an undressed pixel would also collect more background and thus have a larger background-associated noise term. Here, we experimentally demonstrate that such a SWEDA pixel is able to pick out a signal from the background with better SNR when the SBR is low. Additionally, a SWEDA pixel is actually able to detect a fully buried signal $(\mathrm{SNR}<1)$ that a 
conventional sensor and postdetection background subtraction are unable to pick up.

To briefly summarize, SWEDA is effectively a planar and highly compact destructive interferometer. A generic SWEDA consists of a central hole and a set of concentric grooves that are etched into a surface-plasmoncompatible metal layer [see Fig. 1(a)]. There are two transmissive light components through the central hole-the direct light transmission through the hole and the surface-wave (SW)-enabled component. The SW is generated by the incoming background light field interacting with the concentric grooves [5]. This wave is channeled to the central hole and is converted back to a propagating wave. By judicious choice of the central hole diameter, the period of the groove [" $p$ " parameter in Fig. 1(c)] and the spacing between the hole and innermost groove [" $s$ " parameter in Fig. 1(c)], we can arrange for these two components to be matched in amplitude and to be $180^{\circ}$ out of phase with each other. In this way, a uniform zeroincidence background would be efficiently blocked from transmission due to destructive interference. If such a structure is patterned on a sensor pixel, we can expect a strong background to be blocked from interaction with the photosensitive region and background-associated noise would be eliminated [Fig. 1(a)]. A spatially localized signal light field that is confined such that it does not interact with the SWEDA grooves should transmit through the central hole and be detected [Fig. 1(a)]. Figure 1(b) shows the conventional scenario where an undressed pixel array can be used to collect data from multiple pixels, and postdetection background subtraction is then performed.

We fabricated a SWEDA pixel prototype on a commercial CMOS imager sensor (Aptina MT9P031, with a $2.2 \mu \mathrm{m}$ pixel size) with the following procedure. We first spin coated a 150-nm-thick poly(methylmethacrylate) (PMMA)

(a)

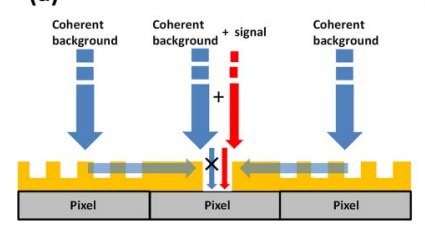

(b)

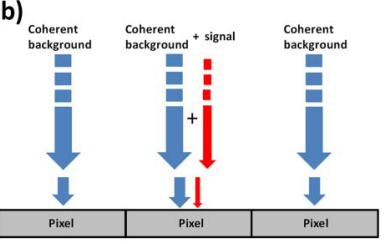

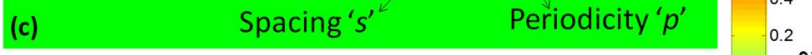

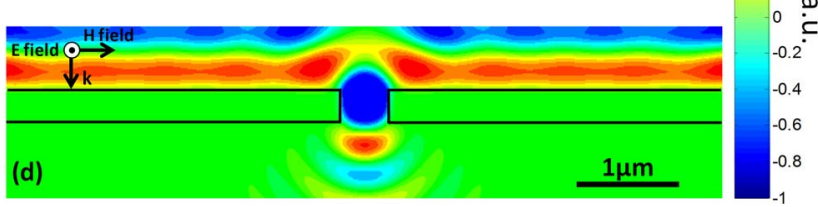

Fig. 1. (Color online) (a) Predetection background suppression scheme of the SWEDA pixel. (b) In conventional schemes, background subtraction is accomplished with the help of a second measurement made by a neighboring pixel. (c) Simulation of the SWEDA structure. Displayed is the real part of the electric field component, equivalent to the time-domain fields at the instant of time when the source phase is zero. (d) Simulation of the single hole. The suppression ratio (transmission of single hole/transmission of SWEDA) is about 8700 in this simulation. layer onto the microlens array of the image sensor to planarize the surface and then coated a $330 \mathrm{~nm}$ gold layer with a thermal evaporator (a $5 \mathrm{~nm}$ chromium layer was used as an adhesion layer). After the metal coating, we used a focused ion beam (FEI Nova 200 dual-beam system) to mill the SWEDA structure. We chose a central hole diameter of $500 \mathrm{~nm}, p=676 \mathrm{~nm}, s=690 \mathrm{~nm}$, depth of groove $=190 \mathrm{~nm}$. Figure 2(a) shows the focus-ion-beam image of a typical structure. In Fig. 2(a), the individual pixel can be barely seen due to the fact that only a thin planarized layer is used and the topology of the surface is not absolutely flat. Figure 2(b) shows the image taken by the sensor under a uniform light illumination with three different wavelengths. The suppression ratio of the SWEDA pixel is optimized for single-wavelength operation at $775 \mathrm{~nm}$. In simulation [Fig. 1(c) and 1(d)], such a SWEDA design is able to suppress background transmission (wavelength $=780 \mathrm{~nm}$ ) with a suppression ratio of $\sim 8700$, compared to an undressed hole of the same size. The measured best suppression ratio was $\sim 1100$ at $775 \mathrm{~nm}$ [Fig. 2(b)]. The deviation between the measured data and the simulation may be due to the nonplanar topology of the surface and imperfect focus-ion-beam milling.

Next, we conducted an experiment to characterize the SNR of the SWEDA pixel under a high background. We used a Ti:sapphire laser (Spectra-Physics Tsunami) as the background illumination source (wavelength of $775 \mathrm{~nm}$ ) and a focused He-Ne laser $(632 \mathrm{~nm})$ to generate a spatially localized signal beam $(10 \times, 0.22 \mathrm{NA}$ objective lens). The signal beam focus had a diameter of $\sim 2 \mu \mathrm{m}$ and was, thus, a match to a single sensor pixel size. We define the SBR as the ratio of the signal intensity to the background intensity. In our experiment, the background intensity was $3.96 \mathrm{~W} / \mathrm{m}^{2}$. Figure 3(a) shows the SNR (mean value/standard deviation of the signal) versus different SBR values. For each data point, we collected 1000 consecutive frames and used this data to calculate the SNR (the exposure time is $5 \mathrm{~ms}$ for each frame). We see that the overall SNR performance of the SWEDA pixel was better than that of a single undressed pixel over a significant SBR range. At the small signal region of Fig. 3(a) the SNR of the SWEDA pixel was higher than the single pixel, because the noise fluctuation of the bright background was the dominant noise term at this (a)

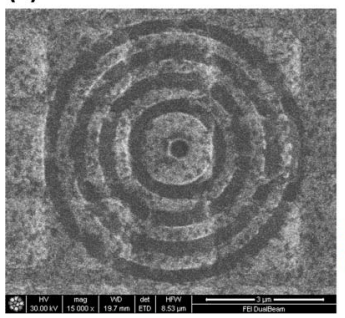

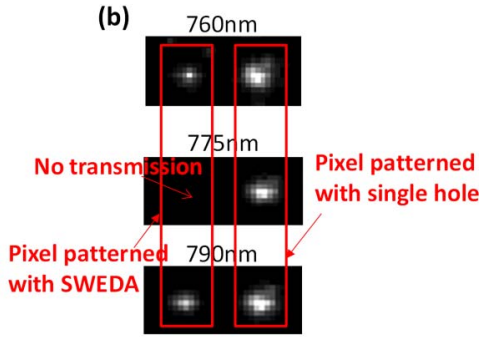

Fig. 2. (Color online) (a) Focus-ion-beam image of the SWEDA pixel (based on Aptina MT9P031 sensor, with a $2.2 \mu \mathrm{m}$ pixel size). (b) Images taken by the sensor under a uniform light illumination with three different wavelengths. The SWEDA pixel is optimized for a single wavelength, and the measured best suppression ratio was $\sim 1100$ at $775 \mathrm{~nm}$. We also note that in Fig. 2(b), the CMOS image sensor was overexposed to clearly show the difference between the SWEDA pixel and the pixel with a simple hole opening. 

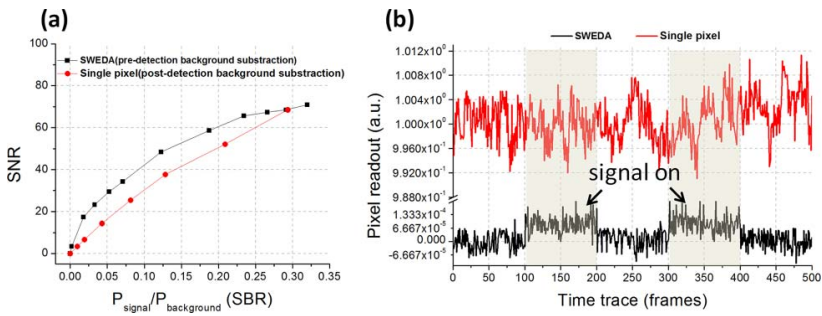

Fig. 3. (Color online) (a) SNR curves versus different SBR values. The background intensity is fixed at $3.96 \mathrm{~W} / \mathrm{m}^{2}$. (b) We used an optical chopper to modulate the input focus signal beam and record the time trace of the SWEDA pixel readout in the black upper curve $(\mathrm{SNR}=2.2, \mathrm{SBR}=0.001)$. The time trace of the single pixel readout is shown in the red lower curve $(\mathrm{SNR}=2.26, \mathrm{SBR}=0.001)$.

region. At the large signal region of Fig. 3(a) the SNR of the single pixel became similar or exceeded that of the SWEDA

pixel, because the noise from the signal itself began to take over. At this point, the much larger photon acceptance area of the single undressed pixel versus the SWEDA pixel (experimentally measured be to a ratio of 22) provided an overriding advantage.

There are two details worth noting at this point. First, although our detection process is not shot-noise limited, the noise term of the energy measured is proportional to the square root of the energy measured, and thus we can expect our measurements to obey the proportionality SNR relationships described in Eqs. (1) and (2). Intrinsic detector noise is not a significant factor in this experiment, as the signal and background power are significantly higher than the measurement threshold of our sensor. Second, the SNR difference does depend on SBR and the background power (basically, we need to specify any two of these three quantities: SBR, background power, or signal power). Based on Eqs. (1) and (2), we can expect the SWEDA pixel to outperform an undressed pixel for low SBR and high background power.

We have, so far, demonstrated improved SNR over a SBR range of the SWEDA pixel. However, perhaps, a more interesting demonstration is the ability of the SWEDA pixel to provide SNR $>1$ for a signal with a very low SBR value, a challenge that has not been met by conventional detection approaches. Here, we used an optical chopper to modulate the input focus signal beam (the optical chopper is like a switch to turn the signal beam on and off at a certain rate) and recorded the time trace of the pixel readout in Fig. 3(b). The control case with a single pixel also shown in Fig. 3(b) for comparison. The SBR value was 0.001 , and the background intensity was
$3.96 \mathrm{~W} / \mathrm{m}^{2}$ for Figs. 3(a) and 3(b). We see that, for the single pixel in Fig. $3(\mathrm{~b})$, the signal was totally masked by the background $(\mathrm{SNR}=0.26)$; yet for the SWEDA pixel in Fig. 3(b), it is well resolvable (SNR = 2.2). Therefore, for the weak signal buried in high background scenarios, our SWEDA pixel approach does provide a better platform than the conventional detection approaches.

At this point, we like to note that the limited photon acceptance area of the SWEDA pixel prototype can potentially be addressed by replacing the central opening with a C-shape aperture [6] to increase light collection efficiency. We also note that the present structure is only optimized for single-wavelength operation. This limitation can be overcome by using more complicated SWEDA-type structures involving multibeam interference that can operate over a broad range of wavelengths.

To summarize, we demonstrate a pixel-level predetection background suppression scheme for increasing the SNR of a conventional image sensor when a high background is present. Because the whole SWEDA pixel size is about $5 \mu \mathrm{m} \times 5 \mu \mathrm{m}$, the packing density can be as high as $10^{6}$ sensing elements per square centimeter. This pixel-level interferometer can potentially be used for protein binding detection [7], highly sensitive optofluidic microscopes [8,9], real-time chemical reaction monitoring, and biosensing $[9,10]$. Some future studies of the SWEDA structure, such as the largest central hole opening limit, the optimal groove profile for SW coupling, and the trade-off between the overall size of the SWEDA and the suppression ratio, are highly desired.

\section{References}

1. R. Narayanaswamy and O. Wolfbeis, Optical Sensors: Industrial, Environmental and Diagnostic Applications (Springer, 2004).

2. G. Konstantatos and E. Sargent, Nature Nanotech. 5, 391 (2010).

3. R. Bhat, N. Panoiu, S. Brueck, and R. Osgood, Opt. Express 16, 4588 (2008).

4. G. Zheng, X. Cui, and C. Yang, Proc. Natl. Acad. Sci. USA 107, 9043 (2010).

5. P. Lalanne and J. Hugonin, Nature Phys. 2, 551 (2006).

6. X. Shi, L. Hesselink, and R. Thornton, Opt. Lett. 28, 1320 (2003).

7. E. Phizicky and S. Fields, Microbiol. Mol. Biol. Rev. 59, 94 (1995).

8. X. Heng, D. Erickson, L. Baugh, Z. Yaqoob, P. Sternberg, D. Psaltis, and C. Yang, Lab Chip 6, 1274 (2006).

9. G. Zheng, Y. M. Wang, and C. Yang, Opt. Express 18, 16499 (2010).

10. N. Lindquist, A. Lesuffleur, H. Im, and S. Oh, Lab Chip 9, 382 (2009). 